\title{
Density Scaling and Virial Theorem
}

\author{
Á. Nagy \\ Department of Theoretical Physics, University of Debrecen, H-4010 Debrecen, Hungary
}

(Dated: January 26, 2015)

\begin{abstract}
The virial theorem, the Levy-Perdew relation and the differential virial theorem are derived for density scaled Kohn-Sham systems. Earlier it was shown that there exists a value of the scaling factor for which the correlation energy disappears and we should treat only exchange for which a simple approximation was proposed. The new Levy-Perdew relation is applied to judge the quality of this approximation.
\end{abstract}




\section{INTRODUCTION}

This paper is dedicated to the memory of Nicholas Handy. He was an outstanding scientist, he had very important contributions to several fields of quantum chemistry. Among others, he emphasized the importance of density scaling [1] in the density functional theory. This paper can be considered as a continuation of his ideas: the derivation of the virial theorem for the scaled energy components.

A kind of scaling, the so-called coordinate scaling has been applied in density functional theory for decades. The functionals expressed with the scaled density $\varrho_{\lambda}(\mathbf{r})=\zeta^{3} \varrho(\lambda \mathbf{r})$ should satisfy several important relations $[2,3]$ that turned to be very useful in constructing and improving approximate functionals.

The density scaling proposed by Chan and Handy [1] is another type of scaling, where the density is multiplied by a positive real number $\zeta$, that is, $\varrho(\mathbf{r}) \rightarrow \zeta \varrho(\mathbf{r})$. Several studies [4-14] utilized it in density functional theory. Zhao, Morrison, and Parr had previously investigated closely related homogeneity relations between the functionals [15-17]. In this paper the virial theorem will be in the focus.

\section{DENSITY SCALING}

In the density functional theory [18] we generally use the Kohn-Sham scheme [19], where the Kohn-Sham equations

$$
\left[-\frac{1}{2} \nabla^{2}+v_{K S}(\mathbf{r})\right] \phi_{i}=\varepsilon_{i} \phi_{i}
$$

are solved with the Kohn-Sham potential

$$
v_{K S}(\mathbf{r})=v(\mathbf{r})+v_{J}(\mathbf{r})+v_{x c}(\mathbf{r})
$$

$\phi_{i}, \varepsilon_{i}, v, v_{J}$ and $v_{x c}$ are the orbitals, orbital energies, the external potential, the classical Coulomb potential and the exchange-correlaton potential, respectively. The total energy can be given by the quantities defined in the Kohn-Sham theory, namely,

$$
E[\varrho]=T_{s}[\varrho]+J[\varrho]+E_{x c}[\varrho]+\int \varrho(\mathbf{r}) v(\mathbf{r}) d \mathbf{r}
$$

where $T_{s}, E_{x c}$ and

$$
J[\varrho]=\frac{1}{2} \int \frac{\varrho\left(\mathbf{r}_{1}\right) \varrho\left(\mathbf{r}_{2}\right)}{\left|\mathbf{r}_{1}-\mathbf{r}_{2}\right|} d \mathbf{r}_{1} d \mathbf{r}_{2}
$$


are the non-interacting kinetic energy, the exchange-correlation energy and the classical Coulomb energy, respectively.

Now, a "scaled" Kohn-Sham system is introduced that corresponds to the scaled density $\varrho_{\zeta}(\mathbf{r})=\varrho(\mathbf{r}) / \zeta$. Due to the scaling the particle number changes, too: $N_{\zeta}=N / \zeta$. As we now generally have noninteger particle numbers, density matrices [1] should be applied in the derivation of the "scaled" Kohn-Sham equation. Then the "scaled" kinetic energy and the scaled density read as

$$
T_{\zeta}[\varrho]=\min _{\zeta \sum_{i}^{M} \lambda_{i}\left|\phi_{\zeta i}\right|^{2} \rightarrow \varrho}\left[-\frac{1}{2} \zeta \sum_{i}^{M} \lambda_{i}\left\langle\phi_{\zeta i}\left|\nabla_{i}^{2}\right| \phi_{\zeta i}\right\rangle\right] .
$$

and

$$
\varrho_{\zeta}(\mathbf{r})=\sum_{i}^{M} \lambda_{i}\left|\phi_{\zeta i}(\mathbf{r})\right|^{2}
$$

where $\lambda_{i}$ are the occupation numbers and $M$ is the number of orbitals with non-zero occupation number. The minimization of the "scaled" kinetic energy leads to the "scaled" Kohn-Sham equations

$$
\left[-\frac{1}{2} \nabla^{2}+v_{\zeta K S}(\mathbf{r})\right] \phi_{\zeta i}=\varepsilon_{\zeta i} \phi_{\zeta i}
$$

where the "scaled" Kohn-Sham potential has the form

$$
v_{\zeta K S}(\mathbf{r})=v(\mathbf{r})+v_{J}(\mathbf{r})+v_{\zeta x c}(\mathbf{r}) .
$$

$v_{\zeta x c}$ is the "scaled" exchange-correlaton potential. The total energy has a new partition:

$$
E[\varrho]=T_{\zeta}[\varrho]+J[\varrho]+E_{\zeta x c}[\varrho]+\int \varrho(\mathbf{r}) v(\mathbf{r}) d \mathbf{r}
$$

where the "scaled" exchange-correlation energy $E_{\zeta x c}[\varrho]$ is defined by Eq. (9) and the functional derivative of $E_{\zeta x c}[\varrho]$ is the "scaled" exchange-correlaton potential $v_{\zeta x c}$. Note that there is no $\zeta$ dependence in the classical Coulomb and external energy parts, only the kinetic energy and the exchange-correlation energy are scaled. It means that the scaling does not change the sum of the kinetic and the exchange-correlation energies. From Eqs. (3) and (9) we arrive at the significant relations

$$
T_{s}[\varrho]+E_{x c}[\varrho]=T_{\zeta}[\varrho]+E_{\zeta x c}[\varrho]
$$


and

$$
\frac{\delta T_{s}[\varrho]}{\delta \varrho}+v_{x c}(\mathbf{r})=\frac{\delta T_{\zeta}[\varrho]}{\delta \varrho}+v_{\zeta x c}(\mathbf{r})
$$

Eq. (10) reflects that the density scaling either increases the kinetic part and decreases the exchange-correlation term or vice-versa.

\section{VIRIAL THEOREM}

The virial theorem of quantum mechanics in case of Coulomb potential in equilibrium molecular geometry has the form

$$
T=-E
$$

where $T$ and $E$ are the kinetic and total energies, respectively. In the Kohn-Sham scheme of the density functional theory it can be written as [2]

$$
T_{s}+T_{c}=-E
$$

as the non-interacting kinetic energy $T_{s}$ differs from the interacting kinetic energy $T$. As the difference $T_{c}$ is positive the inequality $T_{s}<-E$ holds.

Using the "scaled" Kohn-Sham scheme we can write

$$
T_{\zeta}+T_{c \zeta}=-E
$$

instead of Eq. (13).

Now, use the operator $\mathbf{r} \cdot \nabla$ on the "scaled" Kohn-Sham equations (7), multiply by $\lambda_{i} \phi_{\zeta i}^{*}$, integrate and sum for all orbitals. Then we are led to the virial theorem

$$
T_{\zeta}=\frac{1}{2} \int \varrho \mathbf{r} \cdot \nabla v_{K S}(\mathbf{r}) d \mathbf{r}
$$

Using the partition of the "scaled" Kohn-Sham potential (8) and the identities

$$
\begin{gathered}
J=-\int \varrho(\mathbf{r}) \mathbf{r} \cdot \nabla v_{J}(\mathbf{r}) d \mathbf{r} \\
\int \varrho(\mathbf{r}) v(\mathbf{r}) d \mathbf{r}=-\int \varrho(\mathbf{r}) \mathbf{r} \cdot \nabla v(\mathbf{r})
\end{gathered}
$$


in equilibrium, we obtain

$$
2 T_{\zeta}[\varrho]=-J[\varrho]-\int \varrho(\mathbf{r}) v(\mathbf{r}) d \mathbf{r}+\int \varrho(\mathbf{r}) \mathbf{r} \cdot \nabla v_{\zeta x c}(\mathbf{r})
$$

Making use of Eqs. (9) and (14), Eq. (18) leads to the "scaled" Levy-Perdew relation

$$
T_{c \zeta}+E_{\zeta x c}=-\int \varrho \mathbf{r} \cdot \nabla v_{\zeta x c}(\mathbf{r}) d \mathbf{r}
$$

\section{DIFFERENTIAL VIRIAL THEOREM}

The differential virial theorem was derived by Holas and March [20] in the density functional theory. Now it is extended to the "scaled" Kohn-Sham scheme. The "scaled" KohnSham equations (7) can be rewritten as

$$
v_{\zeta K S}-\varepsilon_{\zeta i}=\frac{1}{2} \frac{\nabla^{2} \phi_{\zeta i}^{R e}}{\phi_{\zeta i}^{R e}}=\frac{1}{2} \frac{\nabla^{2} \phi_{\zeta i}^{I m}}{\phi_{\zeta i}^{I m}}
$$

Differentiating Eq. (20) with respect to $x_{\alpha}$, then multiplying with $\left(\phi_{\zeta i}^{R e}\right)^{2}$ and summing for all $i$, then repeating the procedure after replacing $\phi_{\zeta i}^{R e}$ by $\phi_{\zeta i}^{I m}$ and adding the two final equations and integrating, we arrive at the differential virial theorem for the "scaled" quantities

$$
n(\mathbf{r}) \frac{\partial v_{\zeta K S}}{\partial x_{\alpha}}=\frac{1}{4} \frac{\partial}{\partial x_{\alpha}} \nabla^{2} n(\mathbf{r})-2 \sum_{\beta} \frac{\partial}{\partial x_{\beta}} t_{\zeta \alpha \beta},
$$

where $t_{\zeta \alpha \beta}$ is the "scaled" non-interacting kinetic energy density tensor defined by

$$
t_{\zeta \alpha \beta}=\left.\frac{1}{4}\left[\frac{\partial^{2}}{\partial x_{\alpha}^{\prime} \partial x_{\beta}^{\prime \prime}}+\frac{\partial^{2}}{\partial x_{\beta}^{\prime} \partial x_{\alpha}^{\prime \prime}}\right] \gamma_{\zeta}\left(\mathbf{r}^{\prime} ; \mathbf{r}^{\prime \prime}\right)\right|_{\mathbf{r}^{\prime}=\mathbf{r}^{\prime \prime}=\mathbf{r}} .
$$

The "scaled" first-order non-interacting density matrix $\gamma_{\zeta}$ can be expressed with the "scaled" orbitals as

$$
\gamma_{\zeta}\left(\mathbf{r}, \mathbf{r}^{\prime}\right)=\zeta \sum_{i} \lambda_{i} \phi_{\zeta i}^{*}(\mathbf{r}) \phi_{\zeta i}\left(\mathbf{r}^{\prime}\right)
$$

$t_{\zeta \alpha \beta}$ is a real, symmetric tensor. The trace of $t_{\zeta \alpha \beta}$ gives the "scaled" non-interacting kinetic energy density $t_{\zeta}$ that integrates to the "scaled" non-interacting kinetic energy

$$
T_{\zeta}=\int \sum_{\alpha} t_{\zeta \alpha \alpha}(\mathbf{r}) d \mathbf{r}=\int t_{\zeta}(\mathbf{r}) d \mathbf{r} .
$$

The differential virial theorem (21) can be rewritten as

$$
\nabla v_{\zeta K S}=-\mathbf{f}_{\zeta K S}
$$


where the force field $\mathbf{f}_{\zeta K S}$ is defined

$$
\mathbf{f}_{\zeta K S}=\frac{-\frac{1}{4} \nabla \nabla^{2} n(\mathbf{r})+\mathbf{z}_{\zeta K S}}{n(\mathbf{r})} .
$$

The definition of the vector field $\mathbf{z}_{\zeta K S}$

$$
\mathbf{z}_{\zeta K S}=2 \sum_{\beta} \frac{\partial}{\partial x_{\beta}} t_{\zeta \alpha \alpha}
$$

is the divergence of the "scaled" non-interacting kinetic energy density tensor.

The form (25) of the differential virial theorem can be considered as a differential equation for the "scaled" Kohn-Sham potential and can be solved as

$$
v_{\zeta K S}(\mathbf{r})=\int_{\infty}^{\mathbf{r}} \mathbf{r}^{\prime} \cdot \mathbf{f}_{\zeta K S}\left(\mathbf{r}^{\prime}\right)
$$

where $v_{\zeta K S}(\infty)=0$ was utilized. Making use of the partition of the "scaled" Kohn-Sham potential (8), the unknown part of $v_{\zeta K S}$, the "scaled" exchange-correlation potential $v_{\zeta x c}$ can be obtained from Eq. (28). This equation can be used to check the accuracy of an approximate potential.

\section{DISCUSSION AND ILLUSTRATION}

The forms of the virial theorem derived here for the "scaled" system are significant from conceptual and practical points of view. Exact relations and theorems play a very important role in the density functional theory as they proved to be useful in improving the accuracy of approximate energy functionals. In the Kohn-Sham scheme exchange can be treated almost exactly via the optimalized potential (OPM) [21] and the Krieger-Li-Iafrate (KLI) [22] approaches. Only the correlation part of the Kohn-Sham potential should be approximated. It is, however, a very difficult task. As it was shown in [4] density scaling provides an approximation. It turned out that there exists a value of the scaling factor $\left(\zeta_{c}\right)$ for which the "scaled" correlation energy disappears: $E_{c \zeta_{c}}=0$, that is, the "scaled" exchange-correlation energy is equal to the "scaled" exchange energy: $E_{x c \zeta_{c}}=E_{x \zeta_{c}}$. The "scaled" exchange energy $E_{\zeta x}[\varrho]$ is defined by the Hartree-Fock like expression [5]

$$
E_{\zeta x}[\varrho]=-\frac{1}{2} \int \frac{\left|\gamma_{\zeta}\left(\mathbf{r}_{1}, \mathbf{r}_{2}\right)\right|^{2}}{\left|\mathbf{r}_{1}-\mathbf{r}_{2}\right|} d \mathbf{r}_{1} d \mathbf{r}_{2}
$$


where the "scaled" non-interacting one-particle density matrix is given by Eq. (23). Both the OPM and the KLI methods were derived for the "scaled" quantities [5]. The $\zeta$ KLI approximation for the "scaled" exchange potential is

$$
v_{\zeta x}=v_{\zeta S}+\frac{\zeta}{\varrho} \sum_{i=1}^{M}\left\langle\phi_{\zeta i}\left|\lambda_{i} v_{\zeta x}-v_{\zeta x, i}\right| \phi_{\zeta i}\right\rangle\left|\phi_{\zeta i}\right|^{2},
$$

where

$$
v_{\zeta S}=\frac{\zeta}{\varrho} \sum_{i=1}^{M} \phi_{\zeta i}^{*} v_{\zeta x, i} \phi_{\zeta i}
$$

is the "scaled" Slater potential and

$$
v_{\zeta x, i}(\mathbf{r})=\frac{\delta E_{\zeta x}\left[\phi_{\zeta i}\right]}{\phi_{\zeta i} \delta \phi_{\zeta i}^{*}}
$$

is the orbital dependent (Hartree-Fock-like) exchange potential. Note that for $\zeta=1$ Eq. (30) gives the original KLI exchange potential.

The values of the scaling factor $\left(\zeta_{c}\right)$ for which the $\zeta$ KLI total energy equals to the exact energy were calculated for several atoms [5]. It should be emphasized, however, that the "scaled" correlation potential is not zero, though the "scaled" correlation energy disappears. Therefore, the virial of the "scaled" correlation potential is also different from zero. So the "scaled" Levy-Perdew relation (19) cannot be rewritten with the "scaled" exchange potential

$$
T_{\zeta_{c} c}+E_{\zeta_{c} x}+\int \varrho \mathbf{r} \cdot \nabla v_{\zeta_{c} x}(\mathbf{r}) d \mathbf{r} \neq 0
$$

The deviation of the left hand side of the "scaled" Levy-Perdew relation (33) from zero provides a test of the $\zeta$ KLI method. Table I shows the values of the $\zeta_{c}$ for which the $\zeta$ KLI total energy equals to the exact energy (also presented in the Table) and the left hand side of the "scaled" Levy-Perdew relation (33). As it is expected the latter values are not zero, but they are small showing that the $\zeta$ KLI method can be a suitable approximation for several applications.

In conclusion, we can state that density scaling is a very powerful technique. The virial theorem and the differential virial theorem are derived for the scaled system. As there exists a value of the scaling factor for which the correlation energy disappears and the exchange can be treated by a generalized OPM or KLI method, a simple approximation with only one parameter can be generated. The virial theorem is applied to judge the quality of the approximation. 


\section{A. Acknowledgements}

The work is supported by the TAMOP 4.2.2.A-11/1/KONV-2012-0036 project. The project is co-financed by the European Union and the European Social Fund. Grant OTKA No. K 100590 is also gratefully acknowledged.

[1] G. K.-L. Chan and N. C. Handy, Phys. Rev. A 59, 2670 (1999).

[2] M. Levy and J. P. Perdew, Phys. Rev. A 32,2010 (1985).

[3] A. Görling and M. Levy, Phys. Rev. B 47,13105 (1993); Phys. Rev. A 50,196 (1994); Int. J. Quantum. Chem.S. 29, 93(1995).

[4] Á. Nagy, Chem. Phys. Lett. 411492 (2005).

[5] Á. Nagy, J. Chem. Phys. 123044105 (2005).

[6] R. C. Morrison, P. W. Ayers and Á. Nagy, J. Chem. Phys. 126127111 (2007).

[7] P. Eliott, D. Lee, A. Cangi and K. Burke, Phys. Rev. Lett 100256406 (2008).

[8] A. P. Gaiduk, S. K. Chulkov and V. N. Staroverov, J. Chem. Theory Comput. 5699 (2009).

[9] Á. Nagy, J. Phys. B 44035001 (2011).

[10] Á. Nagy, in Progress in Theoretical Chemistry and Physics ed. P. Hoggan, ( Springer, Berlin, 2011) p 185.

[11] Á. Nagy, Phys. Rev. A 84032506 (2011).

[12] P. D. Elkind and V. N. Staroverov, J. Chem. Phys. 136124115 (2012).

[13] E. Fabiano and L. A. Constantin, Phys. Rev. A 87012511 (2013).

[14] Á. Nagy, in Theoretical and Computational Developments in Modern Density Functional Theory ed. A. K. Roy (Nova Science Publishers, NY, USA,2013) p. 189

[15] Q. S. Zhao, R. C. Morrison and R. G. Parr, Phys. Rev. A 50, 2138 (1994).

[16] R. C. Morrison and R. G. Parr, Phys. Rev. A 53, R2198 (1996).

[17] R. G. Parr and S. B. Liu, Chem. Phys. Lett. 276, 164 (1997); 278, 341 (1997); 280, 159 (1997).

[18] P. Hohenberg and W. Kohn, Phys. Rev. 136 B864, 1964.

[19] W. Kohn and L.J. Sham, Phys. Rev. 140 A1133, 1965.

[20] A. Holas and N.H. March, Phys. Rev. A 51, 2040 (1995); Á. Nagy and N.H. March, Phys. 
Rev. A 40, 554 (1989).

[21] R. T. Sharp and G. K. Horton, Phys. Rev. A 30,317(1953); K. Aashamar, T. M. Luke and J. D. Talman, At. Data Nucl. Data Tables 22443 (1978).

[22] J. B. Krieger, Y. Li and G. J. Iafrate, Phys. Lett. A 146,256(1990) ; Phys. Rev. A 45,101(1992).

\section{Table I}

The values of $\zeta_{c}$, the exact total energy and $T_{\zeta_{c} c}+E_{\zeta_{c} x}+\int \varrho \mathbf{r} \cdot \nabla v_{\zeta_{c} x} d \mathbf{r}$ calculated with the $\zeta$ KLI method at $\zeta_{c}$ (in Ry).

\begin{tabular}{|l|c|c|c|}
\hline atom & $\zeta_{c}$ & $E_{\text {exact }}$ & $T_{\zeta_{c} c}+E_{\zeta_{c} x}+\int \varrho \mathbf{r} \cdot \nabla v_{\zeta_{c} x} d \mathbf{r}$ \\
\hline \hline $\mathrm{Be}$ & 1.01004 & -29.334 & -0.012 \\
$\mathrm{~B}$ & 1.00766 & -49.300 & 0.002 \\
$\mathrm{~F}$ & 1.00545 & -199.432 & 0.008 \\
$\mathrm{Ne}$ & 1.00540 & -257.852 & -0.018 \\
$\mathrm{Na}$ & 1.00406 & -324.480 & 0.024 \\
$\mathrm{Mg}$ & 1.00350 & -400.048 & 0.030 \\
$\mathrm{Al}$ & 1.00290 & -484.590 & 0.021 \\
$\mathrm{Cl}$ & 1.00189 & -920.008 & 0.035 \\
$\mathrm{Ar}$ & 1.00191 & -1054.776 & -0.026 \\
\hline
\end{tabular}

